\title{
A case of Silver-Russell syndrome with hemangioma...a new syndrome or just an association
}

\begin{abstract}
Silver-Russell syndrome1 is a heterogeneous disorder universally characterized by short stature and asymmetric body habitus. Most cases of Silver-Russel syndrome are sporadic, with no history of the disorder in their family. Vascular malformation is not described as a part of the syndrome in any of the existing literature even in standard text books. ${ }^{1}$ So presence of venous hemangioma with Silver Russel syndrome phenotype can be proposed as new syndrome or just an association.
\end{abstract}

Keywords: Silver-Russell syndrome, hemangioma, short stature, vascular malformation, anthopometry
Volume 4 Issue 3 - 2018

Joydeep Das,' Jiban Kishna, ${ }^{2}$ Sushanta Bhanja, ${ }^{3}$ Jyoti Kiran, ${ }^{3}$ Abhishek Kr.Tiwari, ${ }^{4}$ Tapan $\mathrm{Kr}$ Biswas ${ }^{4}$

'DNB (Pediatrics), MRCPCH, UK

2Professor of Radiodiagnosis and HOD, Department of Radiodiagnosis, Jagannath Gupta Institute of Medical Science, India

${ }^{3}$ Assistant Professor, Department of Pediatrics, Jagannath Gupta Institute of Medical Science, India

${ }^{4}$ Instructor/Senior Resident, Department of Pediatrics, Jagannath Gupta Institute of Medical Science, India

Correspondence: Joydeep Das, Associate Professor, Deparment of Pediatrics, Jagannath Gupta Institute of Medical Science, Budge Budge, Buita, Kolkata: 700I37, India, Tel 9433076643,Email jddasjoydeep@gmail.com

Received: May 15, 2018 | Published: June 19, 2018

\section{Introduction}

Short stature, triangular facies, fifth finger clinodactyle, prominent forehead with asymmetric body habitus constitutes major features of Silver Russel syndrome. ${ }^{1}$ Vascular malformation, venous or arterial is not a feature in the published phenotype. We report a case of Silver Russel phenotype with venous hemangioma.

\section{Case}

Seven years old male child, born out of non consanguineous parents presented with soft compressible swelling over the upper anterio-medial aspect of left leg around knee joint. Swelling was present since birth as slight blue coloured skin staining but over a span of seven years superficial skin changes to light brown (Figure 1). On examination of other system he has a triangular face (Figure 2), short stature, fifth finger clinodactyle, asymmetric body proportion with hemihypertrophy (Figure 3) of right side suggestive of SilverRussel syndrome. Anthopometry showed height for age was less than 3rd centile (WHO 2006), Rt. lower limb is $2.5 \mathrm{~cm}$ longer then left and Rt. thigh is $1.5 \mathrm{~cm}$ larger (Figure $3 \mathrm{~A}$ ) in circumference at mid thigh then left.

Doppler study of soft tissue swelling showed arterio-venous malformations (Figure 4) mostly venous flow channels suggestive of venous hemangioma with feeding vessels coming from anterior tibeal artery and draining vein is great saphenous vein. There is no change in the bone size of both tibea but localized soft tissue swelling is proportionate to hemangioma. To evaluate presence of arterio-venous malformation in other body sites we did USG whole abdomen, CT angio brain but all are normal.

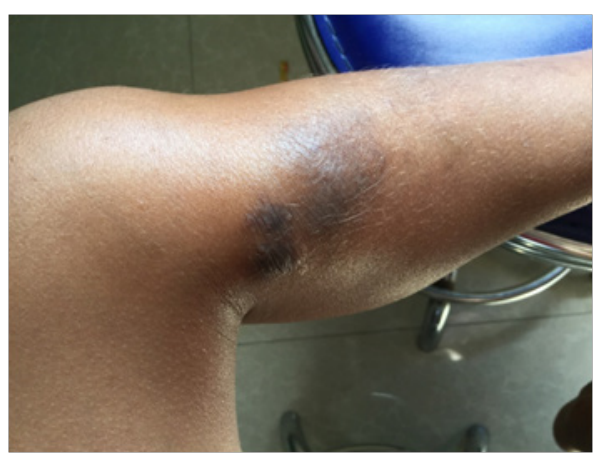

Figure I Slight blue coloured skin staining but over a span of seven years superficial skin changes to light brown.

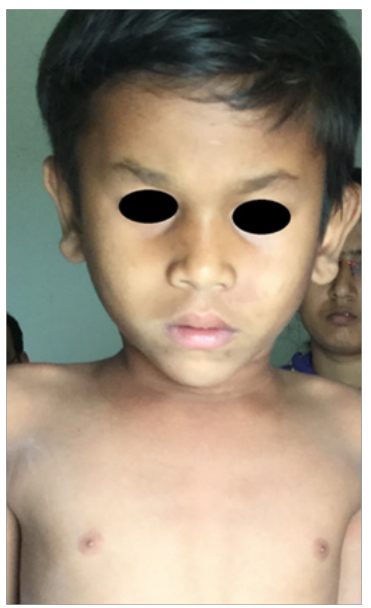

Figure 2 On examination of other system he has a triangular face. 


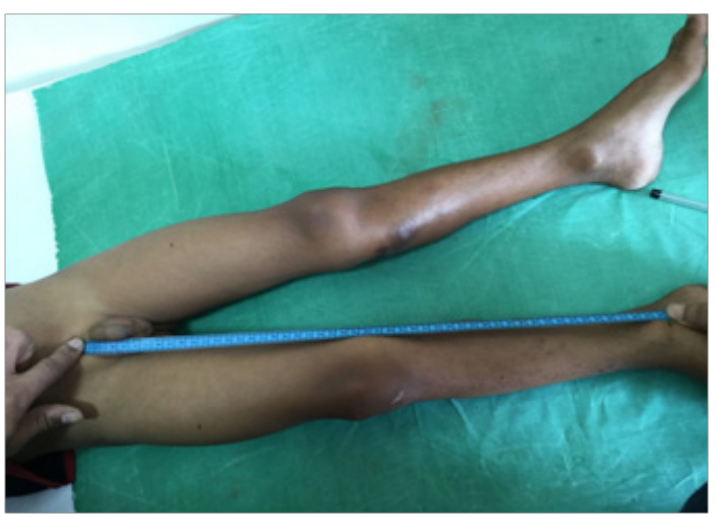

Figure 3 asymmetric body proportion with hemihypertrophy.

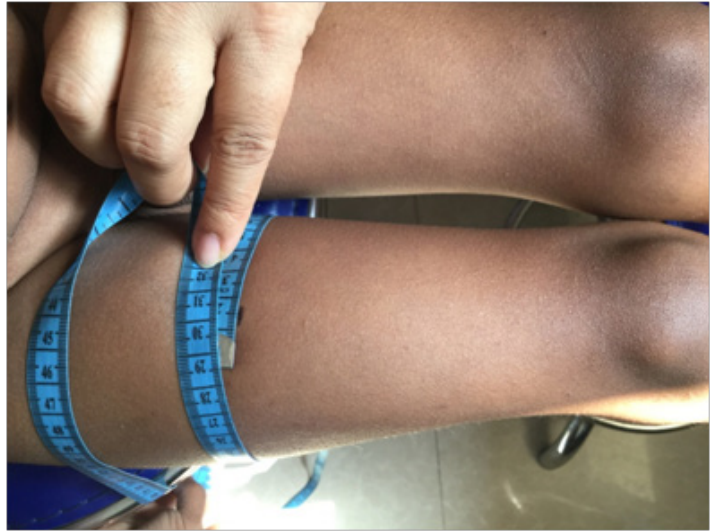

Figure 3A Rt. lower limb is $2.5 \mathrm{~cm}$ longer then left and Rt. thigh is $1.5 \mathrm{~cm}$ larger in circumference at mid thigh then left.

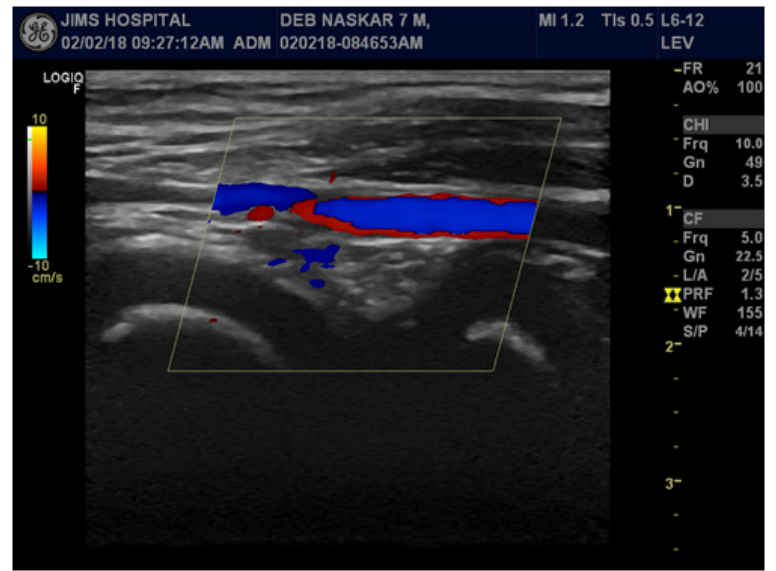

Figure 4 Doppler study of soft tissue swelling showed arterio-venous malformations.

\section{Discussion}

Silver $^{2}$ in 1953 noted a special facies in two low birth weight babies who showed asymetry in the size of the limbs. Russell ${ }^{3}$ in 1954 reported five such babies, some but not all with asymmetry. Silver later summarized the findings in 29 patients. In all cases, the children had been unusually small at birth, in spite of being born at term. The face is triangular with a hypoplastic mandible at the apex. SilverRussell syndrome (SRS) is said to be probably under diagnosed due to the broad range of features.
Hypomethylation of the paternal allele of the H19 gene on chromosome $11 \mathrm{p} 151$ associated with imprinting control region 1 (ICR1), which includes H19 and IGF2, responsible for approximately $50 \%$ of cases. A loss of methylation disrupts the regulation of these genes, which leads to slow growth and the other characteristic features of this disorder. Maternal disomy of chromosome 7 (matUPD7) is another commonly associated genetic anomaly found in this syndrome, occurs when a child inherits both copies of chromosome 7 from the mother, instead of one copy from the mother and one copy from the father. Parent-specific differences in gene expression are caused by a phenomenon called genomic imprinting. Several cases with maternally inherited microduplications or translocations have been familial. Other genetic abnormality documented in this condition are translocation $\mathrm{t}(17 ; 20)$ (q25;q13), ${ }^{4}$ deletion17q22-q24, ${ }^{5}$ and duplication or disruption of chromosome $7 \mathrm{p}^{6}$

Venous malformations are slow-flow vascular malformations due to abnormalities in the development of veins. Venous malformations vary in size and location within the body. When the skin or tissues just under the skin are affected, they appear as a slightly blue-coloured skin stain or swelling. As these are vascular malformations, these are present at birth and grow proportionately to the child.

Price et al. ${ }^{7}$ proposed diagnostic criteria for SRS: (1) birth weight below or equal to $-2 \mathrm{SD}$ from the mean; (2) poor postnatal growth below or equal to $-2 \mathrm{SD}$ from the mean at diagnosis; (3) preservation of occipitofrontal head circumference (OFC); (4) classic facial phenotype; and (5) asymmetry. All the five points are there in our case except venous malformation. So our proposition for a new syndrome or an association is justified.

\section{Acknowledgements}

None.

\section{Conflict of interest}

Author declares that there is no conflict of interest.

\section{References}

1. Russel-Silver Syndrome, P128, Smiths recognizable pattern of human malformation. 7th ed.

2. Silver HK, Kiyasu W, George J, et al. Syndrome of congenital hemihypertrophy, shortness of stature and elevated urinary gonadotrophins. Pediatrics. 1953;12(4):368-376.

3. Russell A. A syndrome of "intra-uterine" dwarfism recognizable at birth with craniofacial dysostosis, disproportionately short arms and other anomalies. Proc R Soc Med. 1954;47(12):1040-1044.

4. Ramírez-Dueñas ML, Medina C, Ocampo-Campos, et al. Severe SilverRussell syndrome and translocation $(17 ; 20)(\mathrm{q} 25 ; \mathrm{q} 13)$. Clin Genet. 1992;41(1):51-53.

5. Eggermann T, Eggermann K, Mergenthaler S, et al. Paternally inherited deletion of CSH1 in a patient with Silver-Russell syndrome. $J$ Med Genet. 1998;35(9):784-786.

6. Monk D, Bentley L, Hitchins M, et al. Chromosome 7p disruptions in Silver Russell syndrome: delineating an imprinted candidate gene region. Hum Genet. 2002;111(4-5):376-387.

7. Price SM, Stanhope R, Garrett C, et al. The spectrum of Silver-Russell syndrome: a clinical and molecular genetic study and new diagnostic criteria. J Med Genet. 1999;36(11):837-842. 